\title{
Eficacia de la punción seca en la discapacidad, umbral de dolor a la presión y movilidad del Síndrome de Dolor Miofascial de hombro en pacientes con Lesión Medular: Estudio piloto, ensayo clínico controlado aleatorizado
}

\author{
Sandra Palacios-Alfonso ${ }^{1}$, César Calvo-Lobo ${ }^{2}$, y Ana Felicitas ${ }^{1}$ \\ ${ }^{1}$ Universidad de León (España); ${ }^{2}$ Universidad Complutense de Madrid (España)
}

\begin{abstract}
El dolor de hombro en pacientes con lesión medular es una de las patologías más prevalentes, en especial por el uso de dispositivos de ayuda para la movilidad, ya que la articulación inicialmente está preparada para otras funciones, y es una articulación muy móvil con poca estabilidad, por lo que suele frecuentemente lesionarse. El objetivo de este estudio es ver si una técnica de punción seca utilizada en estos pacientes es eficaz en la discapacidad (DASH) de dicha muestra. En ese estudio piloto, aleatorizado y controlado simple ciego, se utiliza la técnica de punción seca de entrada y salida rápida de Hong sobre un punto gatillo activo músculo infraespinoso en el grupo experimental y se compara con un grupo control que recibió un tratamiento de aguja placebo. Las variables que se usan en este estudio son: DASHe (Disabilities of the Arm, Shoulder and Hand versión en español), cuestionario McGill del dolor, SPADIe (Shoulder Pain And Disability Index en español) que se evaluaron antes y una semana después de la intervención, y dolor al umbral de presión y rango de movimiento del hombro de rotación interna y aducción horizontal que se evaluaron antes, inmediatamente después y a la semana de la intervención. Se llevó a cabo un análisis estadístico mediante el programa SPSS 22.0, del cual se obtuvieron, tras la homogeneidad de la muestra, que no había diferencias significativas en ninguna de las variables, a excepción del umbral del dolor a la presión tras una semana entre los grupos de estudio. La punción seca utilizada en el síndrome de dolor miofascial de hombro en pacientes con lesión medular parece eficaz para aumentar el umbral del dolor a la semana de tratamiento. Se necesita realizar un estudio con ampliación del tamaño muestral para determinar la efectividad de la intervención.
\end{abstract}

Palabras clave: Dolor de hombro, punción seca, lesión medular, discapacidad.

Efficacy of Dry Needling in Disability, Pressure Pain Threshold and Mobility in Shoulder Myofascial Pain Syndrome in Patients with Spinal Cord Injury: Pilot Study, Randomized Controlled Clinical Trial. Shoulder pain in patients with spinal cord injury is one of the most prevalent pathologies, especially due to the use of assistive devices, since the joint is initially prepared for other functions. It is a hypermobile joint with little stability, so it is often injured. The aim of this study is to prove if dry needling used in these patients is effective in the disability (DASH) of the sample. In this randomized, single-blind, controlled trial, it was used the fast-in and fast-out Hong technique on an active trigger point in infraspinatus muscle within the experimental group and compared with a control group that received a treatment of placebo needle. The variables used in this study are: DASHe (Disabilities of the Arm, Shoulder and Hand version Spanish version), McGill pain questionnaire, SPADIe (Shoulder Pain and Disability Index Spanish version) that were evaluated before and one week after the intervention, and pain pressure threshold (PPT) and range of motion (ROM) of the shoulder of internal rotation and horizontal adduction that were evaluated before, immediately after and a week after the intervention. Statistical analysis was carried out using the SPSS 22.0 program, from which, after the homogeneity of the sample, there were no significant differences in any of the variables, except for the pressure pain threshold after one week between the study groups. Dry needling used in myofascial shoulder pain syndrome in patients with spinal cord injury seems effective in increasing the PPT after one week of treatment. A study with sample expansion is needed to determine the effectiveness of the intervention.

Keywords: Shoulder pain, dry needling, spinal cord injury, disability.

Correspondencia: Sandra Palacios Alfonso. Departamento de Fisioterapia y Enfermería. Campus de Vegazana, s/n. C.P.: 24004. Universidad de León, León (España). E-mail: sandra.palac@gmail.com 
Se considera lesión medular (LM) cualquier perturbación sobre la médula espinal que produzca alteraciones en la sensibilidad, el movimiento y/o la función autónoma (regulación visceral) por debajo del nivel de la lesión (Alcobendas, 2010). El dolor de hombro es una de las patologías más prevalentes en pacientes con LM, tanto en estados más agudos como en crónicos (Jain, Higgins, Katz, y Garshick, 2010).

En pacientes crónicos la causa más común por la que aparece dolor de hombro es el sobreuso de la articulación para una función que inicialmente no está preparada anatómicamente. Se añade que es una articulación muy móvil y con poca estabilidad (Pahys, Mulcahey, Hutchinson, y Betz, 2009) y que se puede encontrar además débil por la lesión si ésta está entre los niveles cervicales. La movilidad con dispositivos de ayuda (sillas de ruedas, bastones, etc.) es algo imprescindible en la gran mayoría de pacientes con lesión medular, por lo que el dolor de hombro puede ser incapacitante para muchas de las actividades diarias (Alm, Saraste, y Norrbrink, 2008).

Por otro lado, se denomina síndrome de dolor miofascial al dolor local o referido ocasionado por los denominados puntos gatillo (PGs). Los puntos gatillo son nódulos hiperirritables dentro de una fibra/banda tensa dentro de un músculo esquelético, que se encuentra acortada de manera permanente, lo que genera disfunciones mecánicas del músculo y dolor (Bron, Dommerholt, Stegenga, Wensing, y Oostendorp, 2011; Bron y Dommerholt, 2012; Chen, Bensamoun, Basford, Thompson, y An, 2007; Gerwin, 2001; Satud, 2007; Simons, 2004, 2008; Simons, Travell, y Simons, 2002).

La activación de un PG se asocia a la sobrecarga muscular debido al abuso mecánico de un músculo, además de por ser mantenido en acortamiento continuado.

Yap (2007) afirma que el dolor crónico está íntimamente relacionado con los puntos gatillo y el síndrome de dolor miofascial, por lo que es necesario su correcto diagnóstico - es decir, realizar una palpación concreta dentro del músculo de una banda tensa y un nódulo, que es especialmente sensible a la presión (Bron et al., 2012; Giamberardino, Affaitati, Fabrizio, y Costantini, 2011; Simons, 2002, 2004, 2008) y posterior tratamiento.

El síndrome de dolor miofascial en la región del hombro aparece relacionado con patologías comunes como tendinopatías o pinzamientos dentro de la articulación (Bron et al., 2011; Pérez-Palomares et al., 2009; Sergienko y Kalichman, 2015), que generan dolor y disfunción en el movimiento del hombro. Por tanto, no es algo exclusivo, si no que puede estar asociado a otras patologías, por lo que su diagnóstico puede ayudar a mejorar la sintomatología en pacientes con dolor de hombro, bien por presencia aislada del síndrome de dolor miofascial, o bien por otras causas comórbidas (Bron et al., 2007; Myburgh, Lauridsen, Larsen, y Hartvigsen, 2011). 


\section{Objetivos}

El objetivo de este estudio es analizar si la técnica de punción seca profunda es eficaz en cambios de la discapacidad (DASH) en pacientes con lesión medular y dolor de hombro.

\section{MÉTODO}

\section{Participantes}

La muestra de estudio son pacientes que han sufrido una lesión medular adquirida, con periodo mínimo de lesión de un año (pacientes crónicos) y un diagnóstico basado en la clasificación ASIA (American Spinal Injury Association) en la que la lesión medular sea:

- Inferior al nivel C5-C6 (para evitar afección directa del hombro por la lesión medular y también del área sensorial, y por ser el nivel más alto en el que se puede utilizar silla de ruedas manual) en casos de tipo ASIA A, B y C.

- Todos los niveles de tratarse de una clasificación ASIA D y E.

Y que además presenten las siguientes características:

a. Criterios de inclusión:

Presentar dolor en el hombro y tener al menos un PG activo en el músculo infraespinoso. Si se encuentran varios PGs dentro de este músculo se procederá a tratar el más sintomático. Para ello se deben encontrar las siguientes características: Banda tensa dentro del músculo infraespinoso, nódulo palpable y que al presionarlo genere dolor referido que el paciente identifique como su dolor.

Sujetos mayores de 18 años: hombres y mujeres.

En caso de utilizar un dispositivo como ayuda a la movilidad podrán participar en el estudio pacientes que usen: ninguno, uno y/o dos bastones, silla de rueda autopropulsada o eléctrica (con motor).

b. Criterios de exclusión:

Lesión ASIA A, B y C por encima del nivel C5-C6 (falta de actividad muscular en el hombro).

Contraindicaciones que derivan de la propia técnica.

Presencia de dolor neuropático.

Inestabilidad medicamentosa.

Otras causas bajo prescripción médica, en las que la punción seca en el hombro esté desaconsejada.

No firmar el consentimiento informado. 


\section{Procedimiento}

Una vez seleccionada la muestra se realizó una aleatorización simple (sorteo con papeletas en sobre cerrado y opaco). El ensayo clínico es un estudio piloto con 8 pacientes $(4+4)$.

Grupo experimental/intervención $(n=4)$ : Recibe un tratamiento de una única intervención basado en la técnica de punción seca en un PG activo del músculo infraespinoso, ya que en relación al hombro es el músculo que se encuentra en más estudios: (Arias-Buría, Fernández-de-Las-Peñas, Palacios-Ceña, Koppenhaver, y Salom-Moreno, 2017; Bron et al., 2011; Calvo-Lobo, Pacheco-da-Costa, Martínez-Martínez, Rodríguez-Sanz, y Cuesta-Álvaro, 2018; Koppenhaver et al., 2016), y éstos afirman que la mayor prevalencia (77\%) en la presencia de PG, cuando existe patología de hombro, se produce en el músculo infraespinoso.

Grupo control $(n=4)$ : Recibe un tratamiento de una única intervención con una aguja placebo validada Streitberger (Streitberger y Kleinhenz, 1998) sobre el área del músculo infraespinoso, cercana al punto gatillo activo.

\section{Instrumentos}

Variables independientes: Se analizaron: sexo, edad, tiempo desde la lesión, hombro doloroso, dominancia, tiempo de dolor de hombro, Spinal Cord Independence Measure (SCIM III). De este último se utilizó el cuestionario validado al español (Aguilar-Rodríguez, Peña-Pachés, Grao-Castellote, Torralba-Collados, y Hervás-Marín, 2015).

Variables dependientes: Se aplicaron los siguientes Cuestionarios:

DASHe, (Disabilities of the Arm, Shoulder and Hand). Adaptación Española (Hervás et al., 2006).

The McGill Pain Questionaire. Adaptación española (Serrano-Atero et al., 2002).

SPADIe (Shoulder Pain and Disability Index). Adaptación española (Luque-Suárez, Rondón-Ramos, Fernández-Sánchez, Roach, y Morales-Asencio, 2016).

Los cuestionarios se aplicaron preintervención y postintervención (una semana posterior al tratamiento aplicado).

Se utilizaron también como variables de medida la algometría (umbral de dolor a la presión) (Hidalgo-Lozano et al., 2011), y el rango articular pasivo (rotación interna y aducción horizontal), siguiendo en este último el protocolo utilizado en Koppenhaver et al. (2016). Ambas mediciones se realizaron preintervención, postintervención inmediatamente después y una semana posterior al tratamiento aplicado. 


\section{Diseño, consideraciones éticas y registro del ensayo}

Este estudio piloto es un ensayo clínico controlado aleatorizado (ECCA) y con un simple ciego (paciente cegado a la intervención mediante un placebo). El estudio además seguirá los criterios CONSORT (Consolidated Standards of Reporting Trials) recomendado para los ensayos clínicos (Schulz, Altman, y Moher, 2010).

Previo a la intervención se solicitó al Comité de Ética de la Universidad, obteniéndose un informe favorable: (ÉTICA-ULE-020-2018). Posteriormente se registró el ensayo en ClinicalTrials.gov., con referencia: (Number Clinical Trial): NCT03709797. Todos los sujetos firmaron un consentimiento informado, previo al inicio de la intervención.

\section{RESULTADOS}

Se reclutaron inicialmente 10 sujetos, siendo 1 excluido por no cumplir los criterios de inclusión (diagnóstico de lesión ASIA B nivel C4).

Figura 1. Diagrama CONSORT
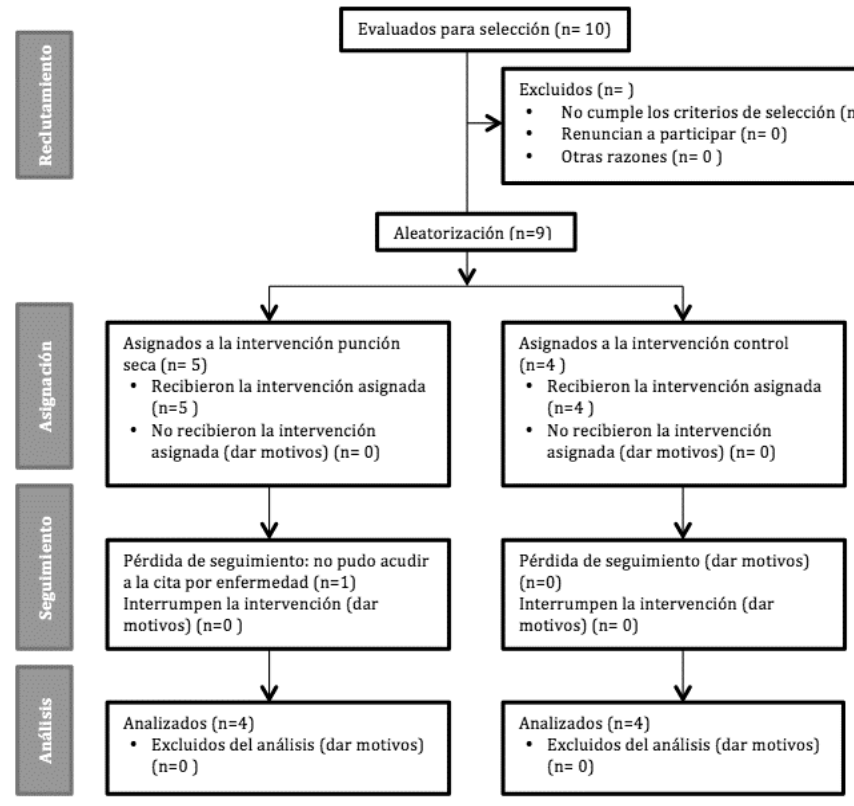

Los 9 sujetos restantes fueron aleatorizados mediante papeletas en sobres cerrados y opacos. 5 fueron asignados al grupo experimental (GE) y 4 al grupo control 
(GC). Durante el seguimiento, un paciente del grupo experimental no puedo asistir a la segunda valoración (tras una semana) por enfermedad. Por tanto, 8 sujetos concluyen el estudio, 4 en GE y 4 en GC (Ver Figura 1).

\section{Datos descriptivos: Homogeneidad de la muestra}

El análisis estadístico inicial de la homogeneidad de la muestra (Tabla 1) se llevó a cabo con los datos demográficos (edad -comprendidas entre 33 y 76 años- y sexo -7 hombres y 1 mujer-) $(n=8)$. También se analizaron tanto las variables independientes como las variables dependientes en el momento inicial. Paralelamente se obtuvieron los mismos datos intragrupo: GE $(n=4)$ y GC $(n=4)$. Estos datos muestran que no hay diferencias significativas $(p \geq 0.05)$ en todas las variables entre los grupos de estudio para ser llevado a cabo su comparación a posteriori.

Tabla 1. Datos descriptivos

\begin{tabular}{|c|c|c|c|c|}
\hline Datos basales de la muestra & $\begin{array}{l}\text { Total } \\
(n=8)\end{array}$ & $\begin{array}{c}\text { Grupo Experimental } \\
(n=4)\end{array}$ & $\begin{array}{c}\text { Grupo Control } \\
(n=4)\end{array}$ & $\begin{array}{c}\text { p-valor } \\
\text { Experimental vs. Control }\end{array}$ \\
\hline \multicolumn{5}{|c|}{ Datos demográficos } \\
\hline Edad (años) & $\begin{array}{l}54.50 \pm 13.72 \\
\quad(33-76)\end{array}$ & $\begin{array}{c}54.50 \pm 17.67 \\
(33-76)\end{array}$ & $\begin{array}{c}54.50 \pm 11.27 \\
(44-70)\end{array}$ & $1.000^{*}$ \\
\hline Sexo: hombre $(\%) /$ mujer $(\%)$ & $\begin{array}{c}7(87.5 \%) / 1 \\
(12.5 \%)\end{array}$ & $3(75 \%) / 1(25 \%)$ & $4(100 \%) / 0(0 \%)$ & $1.000 t$ \\
\hline \multicolumn{5}{|c|}{ Datos clínicos } \\
\hline $\begin{array}{l}\text { ASIA } \\
\text { Clasificación: } \\
\text { n A,B,C }(\%) / \text { n D, E }(\%)\end{array}$ & $\begin{array}{c}7(87.5 \%) / 1 \\
(12.5 \%)\end{array}$ & $4(100 \%) / 0(0 \%)$ & $3(75 \%) / 1(25 \%)$ & $1.000 \div$ \\
\hline $\begin{array}{l}\text { Nivel de lesión } \\
\mathrm{n} \mathrm{C}(\%) / \mathrm{n} \mathrm{D}(\%) / \mathrm{n} \text { L-S (\%) }\end{array}$ & $\begin{array}{c}2(25 \%) / 4(50 \%) / \\
2(25 \%)\end{array}$ & $\begin{array}{c}0(0 \%) / 3(75 \%) / 1 \\
(25 \%)\end{array}$ & $\begin{array}{c}2(50 \%) / 1(25 \%) / \\
1(25 \%)\end{array}$ & $0.223 * *$ \\
\hline Dominancia Dcha (\%)/I (\%) & $8(100 \%) / 0(0 \%)$ & $4(100 \%) / 0(0 \%)$ & $4(100 \%) / 0(0 \%)$ & $1.000 \dagger$ \\
\hline Hombro doloroso Dcha (\%) / I (\%) & $\begin{array}{c}3(37,5 \%) / 5 \\
(62,5 \%)\end{array}$ & $1(25 \%) / 3(75 \%)$ & $2(50 \%) / 2(50 \%)$ & $1.000 t$ \\
\hline $\begin{array}{l}\text { Punción seca previamente NO (\%)/ } \\
\text { SI }(\%)\end{array}$ & $\begin{array}{c}5(62.5 \%) / 3 \\
(37.5 \%)\end{array}$ & $2(50 \%) / 2(50 \%)$ & $3(75 \%) / 1(25 \%)$ & $1.000 t$ \\
\hline Tiempo de lesión (meses) & $\begin{array}{c}44 \pm 123 \\
(18.00-170.00)\end{array}$ & $\begin{array}{c}108.50 \pm 137.25 \\
(18.00-170.00)\end{array}$ & $\begin{array}{c}23.50 \pm 64.25 \\
(18.00-102.00)\end{array}$ & $0.343 \dagger$ \\
\hline $\begin{array}{l}\text { Tiempo desde dolor de hombro } \\
\text { (meses) }\end{array}$ & $\begin{array}{c}19,50 \pm 77 \\
(1.00-170.00) \\
\end{array}$ & $\begin{array}{c}18.00 \pm 126.00 \\
(2.00-170.00) \\
\end{array}$ & $\begin{array}{c}23.50 \pm 77 \\
(1.00-102.00) \\
\end{array}$ & $0.886 \dagger$ \\
\hline SCIMe Autocuidado: $(0-20)$ & $\begin{array}{c}17.50 \pm 3.50 \\
(3.00-20.00)\end{array}$ & $\begin{array}{c}17.50 \pm 4.50 \\
(15.00-20.00)\end{array}$ & $\begin{array}{c}17.50 \pm 11.50 \\
(3.00-18.00)\end{array}$ & $0.686 \dagger$ \\
\hline $\begin{array}{l}\text { SCIMe Respiración y manejo } \\
\text { esfinteriano: }(0-40)\end{array}$ & $\begin{array}{c}30,50 \pm 8,21 \\
(16,00-40,00)\end{array}$ & $\begin{array}{c}31.50 \pm 7.05 \\
(23.00-40.00)\end{array}$ & $\begin{array}{c}29.50 \pm 10.24 \\
(16.00-40.00)\end{array}$ & $0.759^{*}$ \\
\hline SCIMe Movilidad: $(0-40)$ & $\begin{array}{l}20.87 \pm 12.22 \\
(3.00-40.00)\end{array}$ & $\begin{array}{c}22.75 \pm 10.08 \\
(14.00-35.00)\end{array}$ & $\begin{array}{l}19.00 \pm 15.43 \\
(3.00-40.00)\end{array}$ & $0.689 *$ \\
\hline SCIMe Total: $(0-100)$ & $\begin{array}{c}67.12 \pm 24.22 \\
(22.00-97.00)\end{array}$ & $\begin{array}{c}71.75 \pm 19.00 \\
(53.00-95.00)\end{array}$ & $\begin{array}{c}62.50 \pm 3084 \\
(22.00-97.00)\end{array}$ & $0.628^{*}$ \\
\hline McGill Sensorial $(0-10)$ & $\begin{array}{c}5.62 \pm 4.34 \\
(0.00-10.00)\end{array}$ & $\begin{array}{c}5.50 \pm 5.26 \\
(0,00-10,00)\end{array}$ & $\begin{array}{c}5.75 \pm 4.03 \\
(1.00-10.00)\end{array}$ & $0.942 *$ \\
\hline McGill Afectivo $(0-5)$ & $\begin{array}{c}2.12 \pm 2.16 \\
(0.00-5.00) \\
\end{array}$ & $\begin{array}{c}2.50 \pm 2.89 \\
(0.00-5.00) \\
\end{array}$ & $\begin{array}{c}1.75 \pm 1.50 \\
(0.00-3.00) \\
\end{array}$ & $1.000^{*}$ \\
\hline McGill Evaluativo $(0-1)$ & $\begin{array}{c}1.00 \pm 0.00 \\
(0.00-1.00)\end{array}$ & $\begin{array}{c}1.00 \pm 0.00 \\
(0.00-1.00)\end{array}$ & $\begin{array}{c}1.00 \pm 0.00 \\
(0.00-1.00)\end{array}$ & $0.666^{*}$ \\
\hline
\end{tabular}


PALACIOS-ALFONSO et al. Dolor de hombro y discapacidad en pacientes con lesión medular

Tabla 1. Datos descriptivos (continuación)

\begin{tabular}{|c|c|c|c|c|}
\hline Datos basales de la muestra & $\begin{array}{l}\text { Total } \\
(n=8)\end{array}$ & $\begin{array}{c}\text { Grupo Experimental } \\
(n=4)\end{array}$ & $\begin{array}{c}\text { Grupo Control } \\
(n=4)\end{array}$ & $\begin{array}{c}p \text {-valor } \\
\text { Experimental vs. Control }\end{array}$ \\
\hline McGill Miscelánea $(0$ - 4) & $\begin{array}{c}3 \pm 3.75 \\
(0.00-4.00) \\
\end{array}$ & $\begin{array}{c}2.00 \pm 4,00 \\
(0,00-4,00)\end{array}$ & $\begin{array}{c}3.00 \pm 2.25 \\
(1.00-4.00)\end{array}$ & $0.886 \dagger$ \\
\hline McGill Total $(0-20)$ & $\begin{array}{c}11.12 \pm 7.95 \\
(1.00-20.00)\end{array}$ & $\begin{array}{l}11,00 \pm 10,42 \\
(1,00-20,00)\end{array}$ & $\begin{array}{c}11.25 \pm 6.24 \\
(3.00-18.00)\end{array}$ & $0.969^{*}$ \\
\hline McGill PPI (1 - 5) & $\begin{array}{c}2.00 \pm 0.75 \\
(1.00-3.00)\end{array}$ & $\begin{array}{c}2,00 \pm 0.75 \\
(2.00-3.00)\end{array}$ & $\begin{array}{c}2.00 \pm 1.50 \\
(1.00-3.00)\end{array}$ & $0.686 \dagger$ \\
\hline McGill EVA $(0-10)$ & $\begin{array}{c}3.30 \pm 2.78 \\
(2.30-7.20) \\
\end{array}$ & $\begin{array}{c}3.55 \pm 2.55 \\
(2.80-6.10)\end{array}$ & $\begin{array}{c}2.95 \pm 3.80 \\
(2.30-7.20)\end{array}$ & $0.486 \dagger$ \\
\hline DASHe $(0-100 \%)$ & $\begin{array}{l}35.84 \pm 23.76 \\
(5.00-67.59) \\
\end{array}$ & $\begin{array}{c}28.12 \pm 16.56 \\
(13.33-50.00) \\
\end{array}$ & $\begin{array}{l}43.56 \pm 29.01 \\
(5.00-67.59) \\
\end{array}$ & $0.391 *$ \\
\hline SPADIe Intensidad $(0-40)$ & $\begin{array}{c}13.50 \pm 11.75 \\
(11.00-30.00)\end{array}$ & $\begin{array}{c}14.50 \pm 15.50 \\
(11.00-30.00)\end{array}$ & $\begin{array}{c}13.50 \pm 10.75 \\
(11.00-25.00)\end{array}$ & $1.000 \dagger$ \\
\hline SPADIe Dificultad $(0-60)$ & $\begin{array}{l}23,75 \pm 16,17 \\
(9,00-57,00)\end{array}$ & $\begin{array}{c}17.50 \pm 8.18 \\
(9.00-25.00)\end{array}$ & $\begin{array}{l}30.00 \pm 20.94 \\
(9.00-57.00)\end{array}$ & $0.309^{*}$ \\
\hline SPADIe Total $(0-100)$ & $\begin{array}{c}40.38 \pm 19.36 \\
(20.00-70.00)\end{array}$ & $\begin{array}{c}35.00 \pm 16.08 \\
(21.00-55.00)\end{array}$ & $\begin{array}{c}45.75 \pm 23.21 \\
(20.00-70.00)\end{array}$ & $0.475^{*}$ \\
\hline Algometría (kg/cm2) & $\begin{array}{c}3.16 \pm 0.57 \\
(2.43-4.23)\end{array}$ & $\begin{array}{c}3.34 \pm 0.71 \\
(2.50-4.23)\end{array}$ & $\begin{array}{c}2.98 \pm 0.40 \\
(2.43-3.33)\end{array}$ & $0.412^{*}$ \\
\hline Rango Articular RI (grados) & $\begin{array}{c}48.13 \pm 13.24 \\
(30.50-64.50)\end{array}$ & $\begin{array}{c}49.78 \pm 8.23 \\
(41.60-61.00)\end{array}$ & $\begin{array}{c}46.50 \pm 18.28 \\
(30.50-64.50)\end{array}$ & $0.283^{*}$ \\
\hline Rango Articular AH (grados) & $\begin{array}{c}98.63 \pm 16.30 \\
(79.00-118.50)\end{array}$ & $\begin{array}{c}98.75 \pm 13.15 \\
(80.00-110.00) \\
\end{array}$ & $\begin{array}{c}98.50 \pm 21.15 \\
(79.00-118.50)\end{array}$ & $0.985^{*}$ \\
\hline
\end{tabular}

Nota. ASIA: American Spinal Injury Association; C: Cervical, D: Dorsal, L-S: Lumbar-Sacra; Dcha: Derecha, I: Izquierda; SCIMe: Spinal Cord Independence Measure en español; PPI: Present Pain Index, EVA: Escala Visual Analógica; DASHe: Disabilities of the Arm, Shoulder and Hand en español; SPADIe: Shoulder Pain And Disability Index en español; RI: Rotación Interna, AH: Aducción Horizontal. *Se utilizó prueba T-Student para muestras paramétricas: media \pm desviación típica (mínimo - máximo). † Se utilizó la prueba de U-Mann Whitney para muestras no paramétricas: mediana \pm rango intercuartil (mínimo - máximo). ** Se utilizó la prueba de Chi-Cuadrado para frecuencia (\%). $\$$ Se utilizó la prueba exacta de F de Fisher para frecuencia (\%)

Eficacia de tratamiento del grupo experimental versus grupo control a la semana de la intervención

Las variables dependientes que se utilizan en este estudio: McGill, DASHe y SPADIe se compararon antes de la intervención y una semana después de ésta, obteniendo así los datos que se muestran en la tabla 2, del total y de cada uno de los grupos $(p \geq 0.05)$. Estadísticamente no hay diferencias significativas en estos cuestionarios tras una semana desde la intervención.

Eficacia del tratamiento del grupo experimental versus control inmediatamente después y tras una semana de la intervención

Considerando el umbral de dolor a la presión evaluado mediante algometría, el análisis ANOVA de acuerdo a la corrección de Greenhouse-Geisser mostró un efecto significativo para el tiempo $(F=9.153 ; p=0.013)$ y una interacción significativa grupo (2)* tiempo (3) $(F=7.183 ; p=0.023)$ para los cambios mostrados en la tabla 4 . Los análisis post-hoc para las comparaciones múltiples entre los grupos mediante la corrección de Bonferroni mostraron diferencias significativas $(p=0.003)$ generando un mayor aumento del umbral de dolor a la presión a favor del grupo experimental con 
PALACIOS-ALFONSO et al. Dolor de hombro y discapacidad en pacientes con lesión medular

respecto al control, aunque no hubo diferencias entre los grupos para el resto de momentos de medición $(p>0,05)$ (Gráfica 1).

Tabla 2. Comparación de la diferencia de las variables dependientes entre los grupos de estudio a la semana de la intervención

\begin{tabular}{|c|c|c|c|c|}
\hline Variables dependientes & Total $(n=8)$ & $\begin{array}{l}\text { Grupo Experimental } \\
(n=4) \\
\end{array}$ & $\begin{array}{c}\text { Grupo Control } \\
(n=4) \\
\end{array}$ & $\begin{array}{c}p \text { valor } \\
\text { Experimental vs. Control }\end{array}$ \\
\hline McGill Sensorial $(0-10)$ & $\begin{array}{c}0.00 \pm 1 \\
(-7.00-9.00) \\
\end{array}$ & $\begin{array}{l}-0.50 \pm 5.50 \\
(-7.00-0.00)\end{array}$ & $\begin{array}{c}0.00 \pm 7.50 \\
(-1.00-9.00)\end{array}$ & $0.343 \dagger$ \\
\hline McGill Afectivo $(0-5)$ & $\begin{array}{c}0.00 \pm 1 \\
(-4.00-5.00) \\
\end{array}$ & $\begin{array}{c}0.00 \pm 3.00 \\
(-4.00-0.00) \\
\end{array}$ & $\begin{array}{l}-0.50 \pm 4.75 \\
(-1.00-5.00) \\
\end{array}$ & $0.886 \dagger$ \\
\hline McGill Evaluativo $(0-1)$ & $\begin{array}{c}0.00 \pm 0.00 \\
(-1.00-0.00)\end{array}$ & $\begin{array}{c}0.00 \pm 0.75 \\
(-1.00-0.00)\end{array}$ & $\begin{array}{c}0.00 \pm 0.00 \\
(0.00-0.00)\end{array}$ & $0.686 \dagger$ \\
\hline McGill Miscelánea $(0$ - 4) & $\begin{array}{l}-0.25 \pm 1.66 \\
(-3.00-3.00) \\
\end{array}$ & $\begin{array}{c}-0.75 \pm 1.50 \\
(-3.00-0.00)\end{array}$ & $\begin{array}{c}0.25 \pm 1.89 \\
(-1.00-3.00)\end{array}$ & $0.439 *$ \\
\hline Variables dependientes & Total $(n=8)$ & $\begin{array}{l}\text { Grupo Experimental } \\
(n=4)\end{array}$ & $\begin{array}{l}\text { Grupo Control } \\
(n=4)\end{array}$ & $\begin{array}{c}p \text { valor } \\
\text { Experimental vs. Control }\end{array}$ \\
\hline McGill Total $(0-20)$ & $\begin{array}{c}-1.00 \pm 1.75 \\
(-14.00-17.00)\end{array}$ & $\begin{array}{c}-1.00 \pm 10.50 \\
(-14.00-0.00)\end{array}$ & $\begin{array}{c}-1.50 \pm 14.50 \\
(-2.00-17.00) \\
\end{array}$ & $1.000 \dagger$ \\
\hline McGill PPI (1 - 5) & $\begin{array}{c}-0.13 \pm 0.64 \\
(-1.00-1.00) \\
-1.11 \pm 1.49 \\
\end{array}$ & $\begin{array}{c}-0.32 \pm 0.95 \\
(-1.00-1.00) \\
\end{array}$ & $\begin{array}{c}0.00 \pm 0.00 \\
(0.00-0.00)\end{array}$ & $0.638 *$ \\
\hline McGill EVA $(0-10)$ & $(-3.60-1.00)$ & $\begin{array}{c}-1.90-1.53 \\
(-3.60--0.60)\end{array}$ & $\begin{array}{l}-0.32 \pm 1.06 \\
(-1.60-1.00)\end{array}$ & $0.143^{*}$ \\
\hline DASHe $(0-100 \%)$ & $\begin{array}{c}-2.36 \pm 15.69 \\
(-22.50-26.25)\end{array}$ & $\begin{array}{c}-10.37 \pm 8.50 \\
(-22.50--3.34) \\
\end{array}$ & $\begin{array}{c}5.91 \pm 34.13 \\
(-16.76-26.25) \\
\end{array}$ & $0.146^{*}$ \\
\hline SPADIe Intensidad $(0-40)$ & $\begin{array}{c}-3.00 \pm 7.17 \\
(-18.00-5.00) \\
\end{array}$ & $\begin{array}{c}-7.25 \pm 7.27 \\
(-18.00--2.00) \\
\end{array}$ & $\begin{array}{c}1.25 \pm 4.34 \\
(-5.00-5.00) \\
\end{array}$ & $0.092 *$ \\
\hline SPADIe Dificultad $(0-60)$ & $\begin{array}{c}-1.88 \pm 8.11 \\
(-16.00-12.00) \\
\end{array}$ & $\begin{array}{c}-7.00 \pm 6.97 \\
(-16.00-1.00) \\
\end{array}$ & $\begin{array}{c}3.25 \pm 5.90 \\
(-1.00-12.00) \\
\end{array}$ & $0.066^{*}$ \\
\hline SPADIe Total $(0-100)$ & $\begin{array}{c}-4.88 \pm 14.49 \\
(-34.00-15.00) \\
\end{array}$ & $\begin{array}{c}-14.25 \pm 13.42 \\
(-34.00--4.00)\end{array}$ & $\begin{array}{c}4.50 \pm 8.66 \\
(-6.00-15.00)\end{array}$ & $0.057 *$ \\
\hline
\end{tabular}

Nota. PPI: Present Pain Index, EVA: Escala Visual Analógica; DASHe: Disabilities of the Arm, Shoulder and Hand en español; SPADIe: Shoulder Pain And Disability Index en español; RI: Rotación Interna, AH: Aducción Horizontal. *Se utilizó prueba TStudent para muestras paramétricas: media \pm desviación típica (mínimo - máximo). † Se utilizó la prueba de U-Mann Whitney para muestras no paramétricas: mediana \pm rango intercuartil (mínimo - máximo)

Gráfica 1. Cambios en el umbral de dolor a la presión en momento 1 (pre), 2(post) y 3(post1) de grupo experimental y grupo control

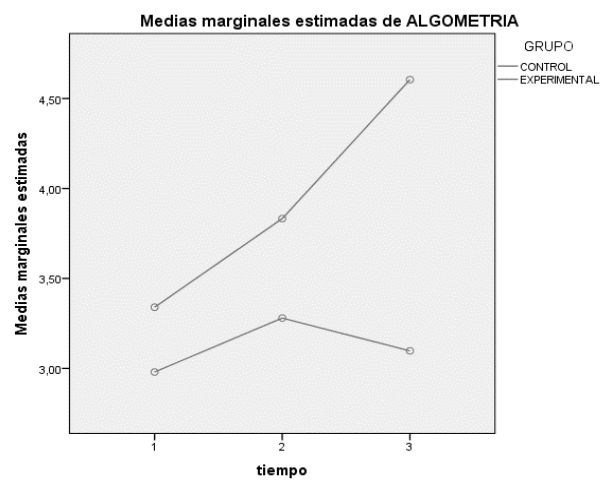

Nota. Gráfico cambios en el umbral de dolor a la presión en momento 1(pre), 2(post) y 3(post1) de grupo experimental y grupo control. Resultados expresados en la unidad $\mathrm{kg} / \mathrm{cm}^{2}$. Obtenido en SPSS 22.0 
Considerando el rango articular en rotación interna, el análisis ANOVA de acuerdo a la corrección de Greenhouse-Geisser no mostró un efecto significativo para el tiempo $(F=3.159 ; p=0.088)$ ni una interacción significativa grupo (2)* tiempo (3) $(F=0.284 ; p=0.731)$ para los cambios mostrados.

Tabla 3. Comparación de la diferencia de Algometría, Rango Articular de Rotación Interna y Aducción Horizontal entre los grupos de estudio tras la intervención y a la semana

\begin{tabular}{|c|c|c|c|c|c|c|}
\hline Variables & Medición & $\begin{array}{c}\text { Total } \\
\text { Media }(D T)\end{array}$ & $\begin{array}{c}\text { Control } \\
\text { Media }(D T)\end{array}$ & $\begin{array}{l}\text { Experimental } \\
\text { Media }(D T)\end{array}$ & $\begin{array}{l}\text { Diferencia de medidas: } \\
\text { Experimental - Control } \\
(\text { LI - LS IC95\%) }\end{array}$ & $\begin{array}{c}p \text { valor experimental } \\
\text { vs. control* }\end{array}$ \\
\hline \multirow{3}{*}{ Algometría } & Pre & $3.16(0.56)$ & $2.98(0.40)$ & $3.34(0.71)$ & $0.36(-0.64-1.36)$ & 0.412 \\
\hline & Post & $3.55(0.69)$ & $3.28(0.60)$ & $3.83(0.73)$ & $0.55(-0.61-1.72)$ & 0.291 \\
\hline & Post 1 & $3.85(0.90)$ & $3.09(0.48)$ & $4.60(0.41)$ & $1.50(0.72-2.29)$ & 0.003 \\
\hline \multirow{3}{*}{$\begin{array}{l}\text { Rango articular } \\
\text { Rotación Interna }\end{array}$} & Pre & $48.13(13.24)$ & $46.50(18.28)$ & $49.77(8.23)$ & $3.27(-21.25-27.80)$ & 0.755 \\
\hline & Post & $55.43(10.92)$ & $51.00(12.74)$ & $59.87(7.98)$ & $8.87(-9.52-27.27)$ & 0.283 \\
\hline & Post 1 & $58.25(11.49)$ & $54.00(15.75)$ & $62.50(3.48)$ & $8.50(-11.24-28.24)$ & 0.333 \\
\hline \multirow{3}{*}{$\begin{array}{l}\text { Rango articular } \\
\text { Aducción Horizontal }\end{array}$} & Pre & $98.62(16.30)$ & $98.50(21.14)$ & $98.75(13.14)$ & $0.25(-30.21-30.71)$ & 0.985 \\
\hline & Post & $100.91(17.27)$ & $98.20(21.61)$ & $103.62(14.47)$ & $5.41(-26.41-37.25)$ & 0.692 \\
\hline & Post 1 & $101.22(14.43)$ & $99.58(18.28)$ & $102.87(12.03)$ & $3.29(-23.48-30.06)$ & 0.774 \\
\hline
\end{tabular}

Nota. DT: desviación Típica; Pre: antes de la intervención, Post: inmediatamente después de la intervención; Post 1: a la semana de la intervención. LI: límite inferior, LS: límite superior, IC95\%: Intervalo de Confianza al 95\%. * $p$-valor del test de comparaciones múltiples pos-hoc entre ambos grupos en los distintos momentos de medición mediante la corrección de Bonferroni

Considerando el rango articular en aducción horizontal, el análisis ANOVA de acuerdo a la corrección de Greenhouse-Geisser no mostró un efecto significativo para el tiempo $(F=1.783 ; p=0.223)$ ni una interacción significativa grupo (2)* tiempo (3) $(F=1.488 ; p=0.269)$ para los cambios mostrados en la tabla 3 .

\section{DISCUSIÓN Y CONCLUSIONES}

Como consideraciones generales cabe reseñar que a nivel general la técnica de punción seca en pacientes con lesión medular en este estudio ha mostrado mejoras en los pacientes del grupo experimental, siendo estadísticamente significativo únicamente en el umbral del dolor a la presión tras una semana postintervención.

En esta investigación encontramos limitaciones relacionadas con el protocolo, como el uso de agujas placebo, ya que diferentes estudios (Braithwaite, Walters, Li, Moseley, y Williams, 2018) refieren que el uso de agujas no tiene una evidencia clara en un simple ciego. El haber utilizado un simple ciego en el diseño experimental. Habría mejorado la calidad metodológica el uso de un doble ciego (diferentes investigadores para realizar la intervención y mediciones). Sin embargo, no ha sido posible realizarlo.

También encontramos limitaciones relacionadas con la muestra, ya que población de estudio se limita a pacientes con lesión medular concreta. Por otro lado, se tiene un pequeño tamaño muestral, así como la posible medicación que toma la muestra de estudio. 
Encontramos limitaciones relacionadas con las variables, debido a la muestra elegida, ya que no existen cuestionarios de mediciones específicas para miembro superior en lesionados medulares.

También limitaciones relacionadas con los objetivos del proyecto, ya que no se han analizado las diferencias intragrupo para mostrar las diferencias en el seguimiento de cada grupo, ya que el objetivo principal planteado se ha centrado en la comparación intergrupos de forma prioritaria.

Por último, como conclusiones del estudio podemos decir que la técnica de punción seca profunda en pacientes con dolor de hombro, que padezcan previamente lesión medular, parece no ser eficaz en (cambios medios tras una semana postintervención): en la discapacidad relacionada con los problemas del miembro superior medida con el Cuestionario DASHe, en el dolor subjetivo medido mediante el McGill Pain Questionaire (McGill), así como tampoco en las actividades funcionales de la vida diaria relacionadas con el dolor del hombro medido con el Cuestionario SPADIe, ni en la algometría inmediatamente después de la intervención.

Tampoco parece ser efectivo en el rango de movimiento en rotación interna y aducción horizontal inmediatamente después de la intervención y tras una semana postintervención.

En cambio, la técnica de punción seca profunda en pacientes con dolor de hombro, que padezcan previamente lesión medular, parece ser eficaz en relación a cambios en la algometría realizada tras una semana postintervención.

\section{REFERENCIAS}

Aguilar-Rodríguez, M., Peña-Pachés, L., Grao-Castellote, C., Torralba-Collados, F., Hervás-Marín, D., y Giner-Pascual, M. (2015) Adaptation and validation of the Spanish self-report version of the Spinal Cord Independence Measure (SCIM III). Spinal Cord, 53(6), 451-454. doi:10.1038/sc.2014.225.

Alcobendas, M. (2010). Conceptos generales sobre el síndrome de lesión medular. En A. Esclarín, Lesión Medular. Enfoque multidisciplinario. Madrid: Editorial Médica Panamericana.

Alm, M., Saraste, H., y Norrbrink, C. (2008). Shoulder pain in persons with thoracic spinal cord injury: prevalence and characteristics. Journal of Rehabilitation Medicine, 40, 277-283.

Arias-Buría, J.L., Fernández-de-Las-Peñas, C., Palacios-Ceña, M., Koppenhaver, S.L., у Salom-Moreno, J. (2017). Exercises and Dry Needling for Subacromial Pain Syndrome: A Randomized Parallel-Group Trial. The Journal of Pain, 18(1), 11-18.

Braithwaite, F.A., Walters, J.L., Li, L.S.K., Moseley, G.L., Williams, M.T., y McEvoy, M.P. (2018). Effectiveness and adequacy of blinding in the moderation of pain outcomes: Systematic review and meta-analyses of dry needling trials. PeerJ-The Journal of Life and Environmental Sciences, 6, 5318. doi:10.7717/peerj.5318

Bron, C., Dommerholt, J.D., Stegenga, B., Wensing, M.A.B., y Oostendorp, R. (2011). High prevalence of shoulder girdle muscles with myofascial trigger points in patients with shoulder pain. BMC Musculoskeletal Disorders, 12, 139. 
Bron, C., Franssen, J., Wensing, M., y Oostendorp, R.A. (2007). Interrater reliability of palpation of myofascial trigger points in three shoulder muscles. The Journal of Manual and Manjpulative Therapy, 15(4), 203-215.

Bron, C., y Dommerholt, J.D. (2012). Etiology of myofascial trigger points. Current Pain and Headache Reports, 16(5), 439-444. doi:10.1007/s11916-012-0289-4

Calvo-Lobo, C., Pacheco-da-Costa, S., Martínez-Martínez, J., Rodríguez-Sanz, D., Cuesta-Álvaro, P., y López-López, D. (2018). Dry Needling on the Infraspinatus Latent and Active Myofascial Trigger Points in Older Adults With Non specific Shoulder Pain: A Randomized Clinical Trial. Journal of Geriatric Physical Therapy, 41(1), 1-13. doi:10.1519/JPT.0000000000000079

Chen, Q., Bensamoun, S., Basford, J.R., Thompson, J.M., y An, K.N. (2007) Identification and quantification of myofascial taut bands with magnetic resonance elastography. Archives of Physical Medicine and Rehabilitation, 88(12), 1658-1661.

Gerwin, R.D. (2001) Classification, epidemiology, and natural history of myofascial pain syndrome. Current Pain Headache Reports, 5(5), 412-420.

Giamberardino, M.A., Affaitati, G., Fabrizio, A., y Costantini, R. (2011). Myofascial pain syndromes and their evaluation. Best Practice and Research: Clinical Rheumatology, 25, 185-198.

Hervás, M.T., Navarro-Collado, M.J., Peiró, S., Rodrigo-Pérez, J.L., López-Matéu, P., y Martínez-Tello, I. (2006) Versión española del cuestionario DASH. Adaptación transcultural, fiabilidad, validez y sensibilidad a los cambios. Medicina Clínica, 127(12), 441-447.

Hidalgo-Lozano, A., Fernández-de-Las-Peñas, C., Díaz-Rodríguez, L., González-Iglesias, J., Palacios-Ceña, F., y Arroyo-Morales, M. (2011). Changes in pain and pressure pain sensitivity after manual treatment of active trigger points in patients with unilateral shoulder impingement: a case series. Journal of Bodywork and Movement Therapies, 15(4), 399-404. doi:10.1016/j.jbmt.2010.12.003

Jain, N.B., Higgins, L.D., Katz, J.N., y Garshick, E. (2010). Association of shoulder pain with the use of mobility devices in persons with chronic spinal cord injury. $P M$ and $R$ : The Journal of Injury, Function, and Rehabilitation, 2(10), 896-900. doi:10.1016/j.pmrj.2010.05.004

Koppenhaver, S., Embry, R., Ciccarello, J., Waltrip, J., Pike, R., Walker M., ... Flynn T. (2016). Effects of dry needling to the symptomatic versus control shoulder in patients with unilateral subacromial pain syndrome. Manual Therapy, 26, 62-69. doi:10.1016/j.math.2016.07.009.

Luque-Suarez, A., Rondon-Ramos, A., Fernández-Sánchez, M.E., Roach, K., y Morales-Asencio, J.M. (2016). Spanish version of SPADI (shoulder pain and disability index) in musculoskeletal shoulder pain: A new 10-items version after confirmatory factor analysis. Health and Quality of Life Outcomes, 14, 32. doi:10.1186/s12955-016-0436-4

Myburgh, C., Lauridsen, H.H., Larsen, A.H., y Hartvigsen, J. (2011). Standardized manual palpation of myofascial trigger points in relation to neck/shoulder pain; the influence of clinical experience on inter-examiner reproducibility. Manual Therapy, 16(2), 136-140. doi:10.1016/j.math.2010.08.002

Pahys, J.M., Mulcahey, M.J., Hutchinson, D., y Betz, R.R. (2009). Scapular stabilization in patients with spinal cord injury. The Journal of Spinal Cord Medicine, 32(4), 389-397.

Pérez-Palomares, S., Oliván-Blázquez, B., Arnal-Burró, A.M., Mayoral del Moral, O., Gaspar-Calvo, E., y De-la-Torre-Beldarraín, M.L. (2009). Contributions of myofascial pain in diagnosis and treatment of shoulder pain. A randomized control trial. BMC Musculoskeletal Disorders, 10, 92. doi:10.1186/1471-2474-10-92 
Schulz, K.F., Altman, D.G., y Moher, D. (2010). CONSORT 2010 Statement: updated guidelines for reporting parallel group randomized trials. British Medical Journal, 4(1), 60-68.

Sergienko, S., y Kalichman, L. (2015). Myofascial origin of shoulder pain: a literature review. Journal of Bodywork and Movement Therapies, 19(1), 91-101. doi:10.1016/j.jbmt.2014.05.004

Serrano-Atero, M.S., Caballero, J., Cañas, A., García-Saura, P.L., Serrano-Álvarez, C., y Prieto, J. (2002). Valoración del dolor (II). Revista de la Sociedad Española del Dolor, 9, 109-121.

Simons, D.G. (2004). Review of enigmatic MTrPs as a common cause of enigmatic musculoskeletal pain and dysfunction. Journal of Electromyography and Kinesiology, 14(1), 95-107.

Simons, D.G. (2008). New Views of Myofascial Trigger Points: Etiology and Diagnosis. Archives of Physical Medicine and Rehabilitation, 89(1), 157-159. doi:10.1016/j.apmr.2007.11.016

Simons, D.G., Travell, J., y Simons, L.S. (2002). Dolor y disfunción miofascial. Tomo 1. El manual de los puntos gatillo. Mitad superior del cuerpo. $2^{\mathrm{a}}$ ed. Madrid, España: Editorial Médica Panamericana.

Streitberger, K., y Kleinhenz, J. (1998) Introducing a placebo needle into acupuncture research. The Lancet, 352(9125), 364-365.

Yap, E.C. (2007). Myofascial pain-an overview. Annals of the Academy of Medicine, Singapore, $36(1), 43-48$.

Recibido: 16 de septiembre de 2019 Recepción Modificaciones: 29 de octubre de 2019 Aceptado: 2 de noviembre de 2019 